\title{
LOS GUAYAQUILEÑOS FRENTE A LA CALIDAD DEL SERVICIO DE LOS RESTAURANTES FRANQUICIADOS INTERNACIONALES
}

\author{
GUAYAQUILEÑOS AGAINST THE QUALITY OF THE SERVICE OF THE \\ INTERNATIONAL FRANCHISED RESTAURANTS
}

\section{CARLOS LUIS TORRES BRIONES' ${ }^{1}$, JUAN ARTURO MOREIRA GARCÍA ${ }^{2}$}

Universidad de Guayaquil.carlos.torres@ug.edu.ec

2 Universidad Católica de Santiago de Guayaquil. juan.moreira@cu.ucsg.edu.ec

RESUMEN

Se determinaron los factores claves para lograr un servicio de calidad dentro de un tipo de negocio muy poco explorado en la ciudad de Guayaquil como lo son los restaurantes franquiciados internacionales. Para poder cumplir este objetivo fue necesario aplicar una investigación cuantitativa que ayude a determinar las variables de la calidad de servicio que influyen en la decisión de consumo de los guayaquileños al momento de elegir un restaurante franquiciado internacional, las cuales podrían llegar a transformarse en factores capaces de conseguir la tan anhelada fidelización del cliente.Se escogió el cuestionario estructurado como herramienta de investigación de mercados, y se encuestaron a un total de 400 personas entre hombres y mujeres de 18 a 40 años de la ciudad de Guayaquil, incluyendo zonas periféricas como la vía a la costa y la vía a Samborondón. En un principio se procedió a determinar si la comida era el factor más importante para los guayaquileños al momento de elegir un restaurante franquiciado internacional. Una vez que se confirmó ese aspecto, se procedió a evaluar una serie de variables relacionadas con las instalaciones, accesibilidad, personal, ambiente, comida y consistencia y honestidad de los restaurantes franquiciados internacionales donde se pudo constatar que los factores claves de éxito para alcanzar la calidad en el servicio son la limpieza, higiene y temperatura agradable del local, las alternativas de pago, el trato cordial del personal de servicio, el sabor de la comida y el tiempo de espera de los pedidos.

PALABRAS CLAVE: servicio, restaurante, internacional, franquicia, marketing.
ABSTRACT

This research defined some of the key success factors to achieve a high quality service in an international franchised restaurant in Guayaquil. In order to reach this goal, it was necessary a quantitative research, which helped to state the variables that influence the consumption decision of people from Guayaquil at the moment of choosing an international franchised restaurant; it is important to mention that these variables could become the most significant factors to obtain the costumer loyalty. A structured questionnaire was chosen as one of the main tools for this market research; and it was used to poll 400 men and women, from 18 to 40 years old from Guayaquil, including the peripheral zones, such as vía a la costa, and vía a Samborondón. At the beginning, it was important to know if food was the key factor for consumers from Guayaquil when they have to choose an international franchised restaurant. Once this factor was defined, many variables were evaluated, such as the facilities, the access, the honesty of employees, the ambience, and the food of international franchised restaurant in Guayaquil. With this research, it was possible to note that the key success factors to achieve a high quality service in this kind of business were the cleanliness of the restaurant, the ambience inside it, the payment methods, the pleasant treatment of service personnel, the flavor of the food, and the waiting time to get the order.

KEYWORDS: service, quality, restaurant, international, franchise, marketing. 


\section{INTRODUCCIÓN}

El presente artículo tiene como base una investigación descriptiva que permite conocer las variables o factores que influyen en la percepción de la calidad de servicio de los Guayaquileños que asisten a restaurantes franquiciados internacionales.

Uno de los esquemas más utilizados en el servicio al cliente es la Cadena de Servicio- Utilidad de los docentes investigadores de la Universidad de Harvard, James ames Heskett y Leonard Schlesinger, referencia quienes establecieron que la lealtad de los clientes es posible si existe un alto nivel de satisfacción en los empleados, ya que esto mejora su nivel de productividad.

Según datos del Instituto nacional de estadística y censos del Ecuador (INEC), en su último censo realizado en el año 2010, el comercio está considerado como la actividad preferida de los guayaquileños. El estudio reveló que Guayaquil tiene el $17.4 \%$ de los establecimientos económicos a nivel nacional. Dentro de estas estadísticas se encuentran los negocios de expendio de alimentos, incluyendo las franquicias de restaurantes internacionales.

En el Ecuador, el sistema de franquicias se inició en 1967 con la llegada de una compañía norteamericana de lavado en seco, la cual en la actualidad cuenta con más de 150 locales en todo el país. Luego llegaron empresas como Kentucky Fried Chicken en 1975, Pizza Hut en el año 1982, Tropi Burguer en 1986, Mc. Donalds en 1997, entre otras, dando la iniciativa a la llegada de otras cadenas no solo de locales de comidas rápidas sino también de restaurantes internacionales.

Palomeque y Ramírez (2012) en una investigación realizada concluyen que las franquicias gastronómicas han demostrado tener un impacto considerable dentro del mercado, y son considerados negocios rentables y de bajo riesgo por parte de los ecuatorianos.

Sin embargo, a pesar del gran impacto antes mencionado de los restaurantes franquiciados internacionales en el mercado ecuatoriano, han existido marcas que han tenido que cerrar sus puertas en el país, como es el caso de la mundialmente reconocida franquicia Hooters, que en noviembre de 2012 dejó de funcionar en la ciudad de Guayaquil después de casi dos años de operaciones. Aunque muchos fueron los rumores acerca de los motivos que causaron el cierre de este restaurante, resulta imposible determinar las razones específicas para tal decisión, y peor aún, el grado de incidencia que tuvo la calidad de su servicio.
Esta investigación tiene como objetivo determinar el grado importancia que tiene la calidad del servicio como factor clave para la fidelización de clientes en los restaurantes franquiciados internacionales de Guayaquil.

\section{RESULTADOS}

En la primera pregunta fue, donde de manera general, se quiso conocer cuáles son los restaurantes franquiciados que suelen visitar con frecuencia los encuestados. El restaurante Friday's fue el local que mayor porcentaje seguido por Chili's y Tony Roma's con porcentajes muy similares, dejando en último lugar a la franquicia ecuatoriana Wingers. Es importante recalcar que los guayaquileños prefieren los restaurantes internacionales frente a un franquiciado local, y los visitan en su mayoría acompañados de amigos, familiares y parejas. El 50\% de los encuestados refirieron que la frecuencia de visita a este tipo de negocios es una vez cada dos meses; es decir ,que como promedio los consumidores acuden a los restaurantes franquiciados internaciones seis veces al año.

Para conocer cuáles aspectos eran determinantes en el momento de elegir el restaurante de su preferencia se le presentó a los encuestados seis opciones, obteniendo los resultados mostados en la tabla 1.

\begin{tabular}{|c|c|c|}
\hline ASPECTOS DETERMINANTES & $\begin{array}{l}\text { NÚMERO DE } \\
\text { RESPUESTAS }\end{array}$ & PORCENTAJE \\
\hline Instalaciones & 110 & $9 \%$ \\
\hline Accesibilidad & 81 & $7 \%$ \\
\hline Atención del personal & 153 & $13 \%$ \\
\hline Ambiente & 281 & $24 \%$ \\
\hline Comida & 343 & $29 \%$ \\
\hline \multirow[t]{2}{*}{ Cumplimiento y buen servicio } & 209 & $18 \%$ \\
\hline & 1177 & $100 \%$ \\
\hline
\end{tabular}

Así mismo la figura 1 muestra gráficamente los factores anteriormente mencionados.

Mediante esta pregunta se pudo conocer cuáles son los principales aspectos que considera el consumidor en el momento de la elección de su restaurante de preferencia, siendo una o varias las alternativas que podía elegir el encuestado en su respuesta.

Los tres aspectos de preferencia más señalados por los encuestados al momento de visitar un restaurante fueron la calidad de la comida, el ambiente en segundo lugar, y finalmente el 
cumplimiento y buen servicio. En esta distribución, se ubica la comida como el principal aspecto que atrae o motiva al cliente a la hora de elegir su restaurante de preferencia.

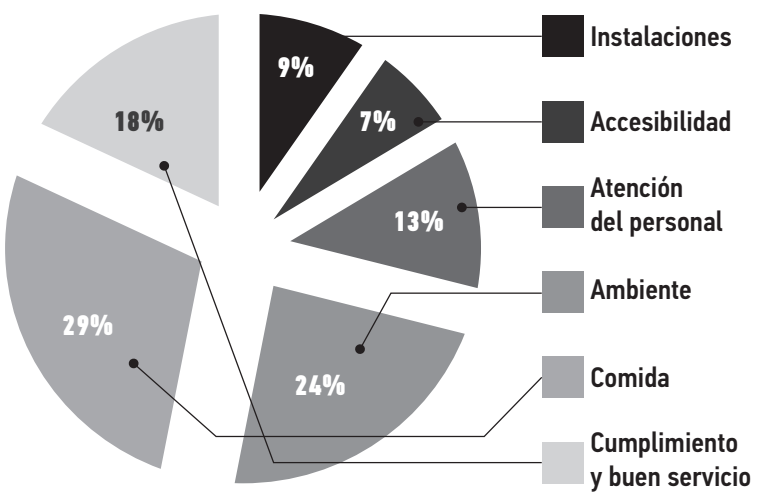

Figura 1. Aspectos determinantes al momento de elegir el restaurante de supreferencia.

Luego de conocer los aspectos más significativos en el momento de elegir un restaurante franquiciado internacional, se procedió a presentar una serie de variables segmentadas en seis grupos, con el fin de determinar cuáles eran los factores más importantes de la calidad del servicio. Estas variables fueron agrupadas según aspectos considerados importantes, tales como instalaciones del restaurante, la accesibilidad, el personal de atención al cliente, el ambiente del local, la comida y los productos que ofrece, y la honestidad y consistencia que debe brindar considerando que se tratan de franquicias que manejan estándares similares de servicio.

Estas variables fueron determinadas a través de la aplicación previa de la observación directa como herramienta de investigación en cuatro de las cadenas de restaurantes franquiciados internacionales de la ciudad de Guayaquil. Para poder medir la relevancia de cada una de las variables, se pidió a los encuestados que califiquen cada una de ellas según su grado de importancia, siendo cinco la calificación más alta y uno la más baja. A continuación en la tabla 2 y figura2, se muestran los resultados de la encuesta realizada a las 400 personas.

Respecto a las instalaciones de los restaurantes, las personas consideran que el aspecto más importante es la limpieza y la higiene que se mantiene en el local, lo cual debe apreciarse desde la entrada, la mesa y los baños. La comodidad es otro factor que los clientes consideran importante, sobretodo en lo que se refiere a la disponibilidad suficiente de espacios. Dentro de los aspectos internos y externos del restaurante, el menos importante para cliente es el externo, es decir que su fachada no tiene mucha relevancia, mientras que el diseño, la arquitectura y los colores del interior del local son más interesantes para el cliente.

$\begin{aligned} & \text { TABLA 2. RESUMEN DE LAS VARIABLES RELACIONADAS A } \\
& \text { INSTALACIONES }\end{aligned}$
\begin{tabular}{lll}
\hline VARIABLE & PROMEDIO & RESULTADO \\
\hline Aspecto Interno & 3,63 & Muy Importante \\
\hline Aspecto Externo & 3,24 & Importante \\
\hline Comodidad & 3,97 & Muy Importante \\
\hline Limpieza e Higiene & 4,40 & Muy Importante \\
\hline
\end{tabular}

Instalaciones

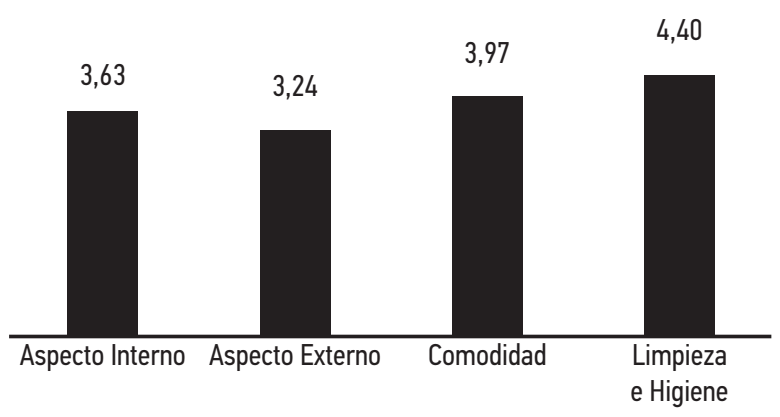

Figura 2. Resumen de las variables relacionadas a instalaciones.

En el grupo de variables relacionadas a la accesibilidad de los restaurantes, se obtuvieron los resultados mostrados en la tabla 3.

\section{TABLA 3. RESUMEN DE LAS VARIABLES RELACIONADAS A ACCESIBILIDAD}

\begin{tabular}{lll}
\hline VARIABLE & PROMEDIO & RESULTADO \\
\hline Estacionamiento & 3,05 & Importante \\
\hline Ubicación & 2,96 & Importante \\
\hline Alternativa de Pago & 3,52 & Muy Importante \\
\hline
\end{tabular}

En la figura 3 se muestra de forma gráfica las variables relacionadas a la accesibilidad.

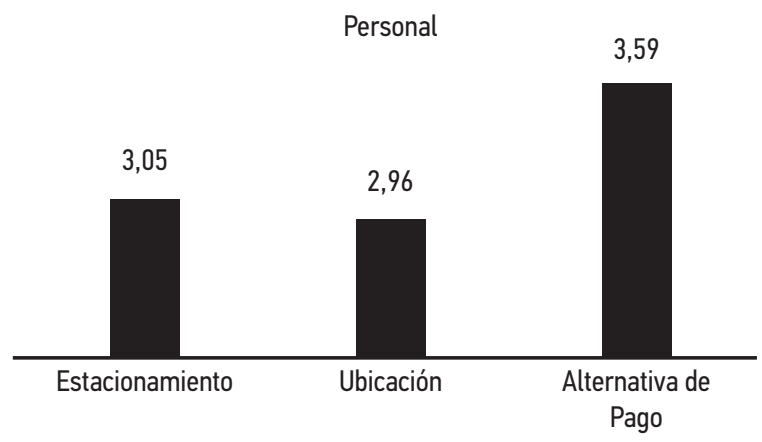

Figura 3. Resumen de las variables relacionadas a accesibilidad. 
Con respecto a la accesibilidad que ofrecen estos locales, los encuestados consideran que la alternativa de pago es el factor más importante en esta categoría, por tal razón los restaurantes ofrecen a su clientela dos formas para cancelar las cuentas que son efectivo o crédito. El factor con menos importancia fue la ubicación, es decir que para el cliente no es importante donde se encuentre el local, ya que si tienen ganas de visitar el restaurante no tendrán ningún inconveniente en movilizarse hasta el mismo.

Una vez determinados las variables de accesibilidad más importantes se procedió a analizar las de personal:

\begin{tabular}{lll}
\hline TABLA 4.RESUMEN & DE LAS VARIABLES RELACIONADAS AL PERSONAL \\
\hline VARIABLE & PROMEDIO & RESULTADO \\
\hline Recepción & 3,98 & Muy Importante \\
\hline Competencia & 3,45 & Importante \\
\hline Presentación Física & 3,85 & Muy Importante \\
\hline Rapidez & 4,37 & Muy Importante \\
\hline Trato Cordial & 4,52 & Lo más importante \\
\hline
\end{tabular}

El resumen de las variables relacionadas al personal así como su nivel de importancia se muestra en la figura 4.

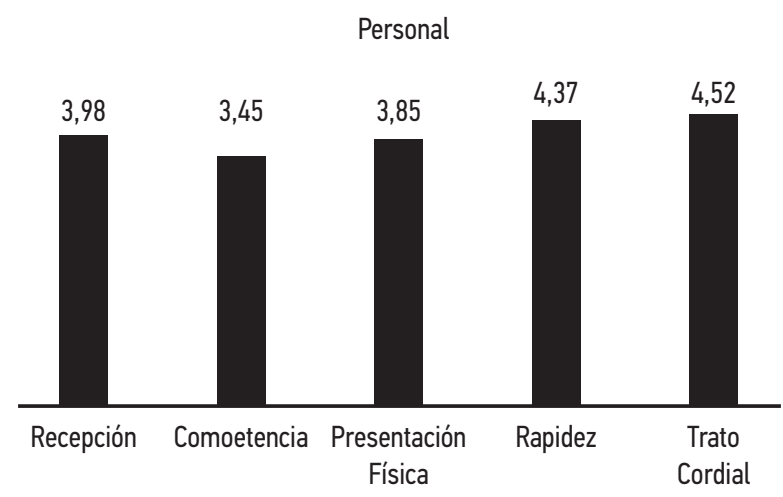

Figura 4. Resumen de las variables relacionadas al personal.

La variable de personal se refiere al grupo de personas que están atentas al requerimiento del cliente, que puede ser el mesero, la anfitriona, el bartender, entre otros. En este aspecto, el encuestado ha decidido que lo más importante en el personal es su trato cordial, es decir la amabilidad, la cordialidad y el respeto que se le ofrece al cliente. Como segundo factor importante resultó la rapidez del personal. Como factores menos importantes resultaron la presencia física y la competencia, es decir que su apariencia o el conocimiento que se tenga sobre la carta del menú no son factores gravitantes para el cliente.
Por otra parte, las variables relacionadas con el ambiente del local se comportaron según lo muestra la tabla y figura 5 .

\begin{tabular}{lll}
\hline TABLA 5. RESUMEN DE LAS VARIABLES RELACIONADAS AL AMBIENTE \\
\begin{tabular}{|lll}
\hline VARIABLE & PROMEDIO & RESULTADO \\
\hline Entorno Audiovisual & 3,12 & Importante \\
\hline Iluminación & 2,87 & Importante \\
\hline Aroma & 3,53 & Muy Importante \\
\hline Temperatura & 3,97 & Muy Importante \\
\hline
\end{tabular}
\end{tabular}

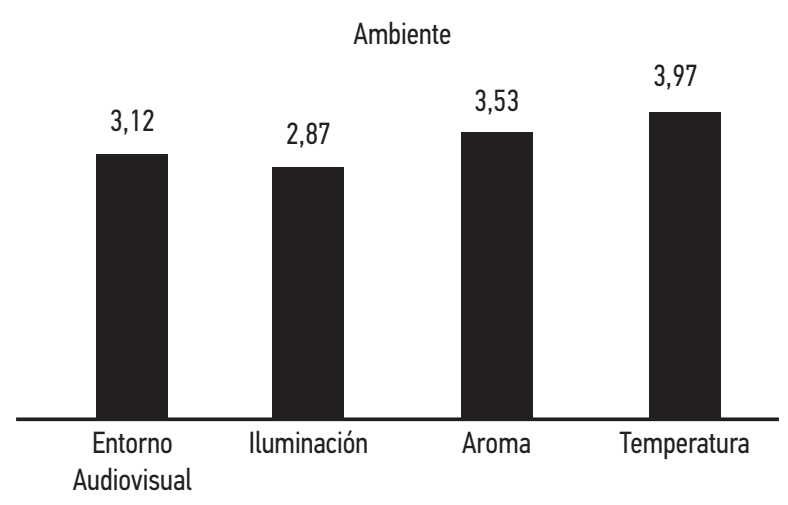

Figura 5. Resumen de las variables relacionadas al ambiente.

En las variables del ambiente, los factores más importantes para el cliente resultaron el aroma y la temperatura, es decir que el cliente espera que el local tenga una adecuada climatización y que se perciba una buena fragancia en el entorno. El factor que menos resulta importante es la iluminación.

En las variables relacionadas a la comida que ofrece el restaurante, se obtuvieron los resultados mostrados a continuación en la tabla 6 , asi como su representación gráfica en la figura 6 .

\section{TABLA 6. RESUMEN DE LAS VARIABLES RELACIONADAS A LA COMIDA}

\begin{tabular}{lll}
\hline VARIABLE & PROMEDIO & RESULTADO \\
\hline Sabor & 4,85 & Lo más importante \\
\hline Olor & 4,24 & Muy Importante \\
\hline Variedad & 3,90 & Muy Importante \\
\hline Presentación Visual & 4,34 & Muy Importante \\
\hline Higiene & 4,73 & Lo más importante \\
\hline Frescura & 4,79 & Lo más importante \\
\hline Temperatura & 4,78 & Lo más importante \\
\hline
\end{tabular}

Los factores más importantes dentro de las variables relacionadas a la comida fueron la higiene, frescura, y temperatura de los alimentos, los tres con un promedio similar. El factor menos atractivo resultó la variedad de la comida, 
ya que probablemente el cliente prefiere calidad en los platos de comida antes de la cantidad de opciones.

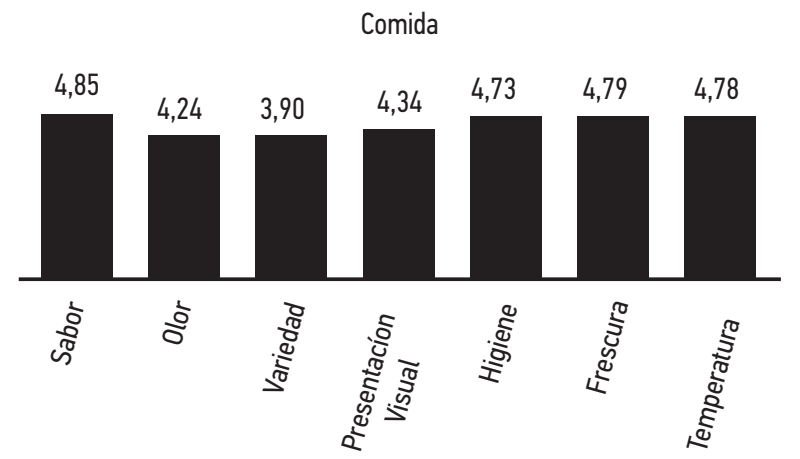

Figura 6. Resumen de las variables relacionadas a la comida.

Finalmente, los resultados de las variables relacionadas a la consistencia y honestidad fueron los mostrados a continuación, en la tabla 7.

\begin{tabular}{|c|c|c|}
\hline VARIABLE & PROMEDIO & RESULTADO \\
\hline Servicio estandarizado & 3,22 & Importante \\
\hline Prestigio & 2,87 & Importante \\
\hline Cumplimiento & 3,57 & Muy importante \\
\hline Atención a quejas & 4,06 & Muy importante \\
\hline Tiempo de espera & 4,39 & Muy importante \\
\hline
\end{tabular}

En la figura 7 se muestra gráficamente la importancia de cada una de las variables relacionadas a la consistencia y honestidad.

\section{Consistencia y Honestidad}

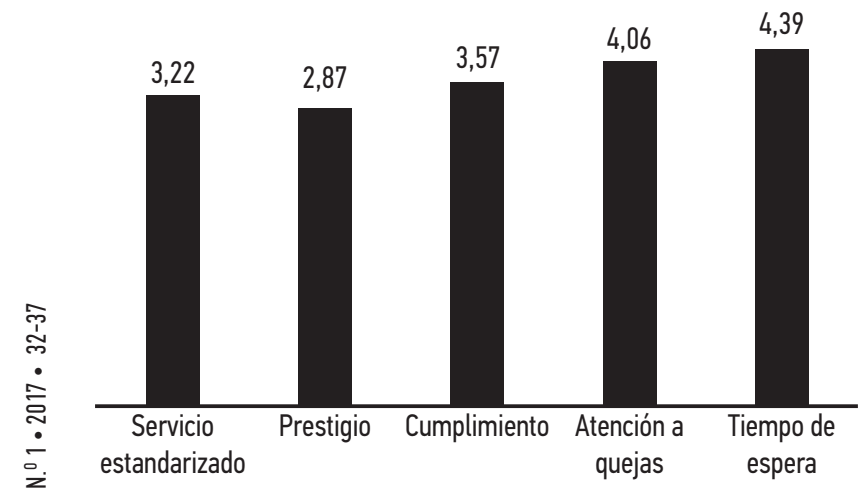

Figura 7. Resumen de las variables relacionadas a la consistencia y honestidad.

Dentro de las variables de consistencia y honestidad, para el cliente son muy importantes los factores de cumplimiento, atención de quejas y tiempo de espera, siendo la última respuesta la de mayor puntuación, es decir el cliente espera que en cualquier local que visite, independientemente de su ubicación, se cumpla con los tiempos estipulados por la propia franquicia. El factor que le resultó menos importante al cliente fue el prestigio que proyecta la marca del local.

Antes de finalizar la investigación, se le preguntó a los encuestados si consideraban que el servicio es un factor determinante para su fidelización con los restaurantes franquiciados internacionales y el 90\% de ellos respondió que sí, lo que demuestra que dentro de este tipo de negocios no solo es importante ofrecer un producto de calidad sino también lograr que los clientes puedan tener una experiencia agradable dentro de los locales.

\section{DISCUSIÓN DE RESULTADOS}

Con los resultados obtenidos es posible definir que los guayaquileños prefieren ante todo la limpieza del restaurante franquiciado y un ambiente con temperatura agradable para poder departir con las personas con las que asiste al lugar, sin importar su ubicación, ya que ellos están dispuestos a movilizarse hasta el local, si la comida les parece buena. Se pudo determinar también que para ellos es importante que los restaurantes acepten tarjetas de crédito o débito, y que la atención del personal sea cordial y capaz de cumplir con los tiempos de espera establecidos por cada franquicia.

Con los datos expuestos, el presente trabajo es de suma importancia ya que hasta el momento no existe ningún estudio sobre la calidad del servicio de los restaurantes franquiciados internacionales en la ciudad de Guayaquil, por lo que será un aporte de primera mano, que permitirá a los dueños, a los administradores y a los encargados de marketing, saber realmente la importancia del servicio al cliente y sus repercusiones en la fidelización de sus marcas.

Los resultados de esta investigación por otro lado, han logrado determinar cuáles son los factores claves que se deben considerar para un tipo de servicio en específico como lo es el de los restaurantes franquiciados.

En la actualidad no existe una investigación realizada sobre la calidad del servicio al cliente como clave del éxito en este tipo de negocios en Guayaquil, por lo tanto se sugiere que se tome en cuenta la siguiente propuesta que incluye los factores claves para un servicio de calidad dentro de un restaurante franquiciados internacional a medida que el cliente ingresa al mismo. 


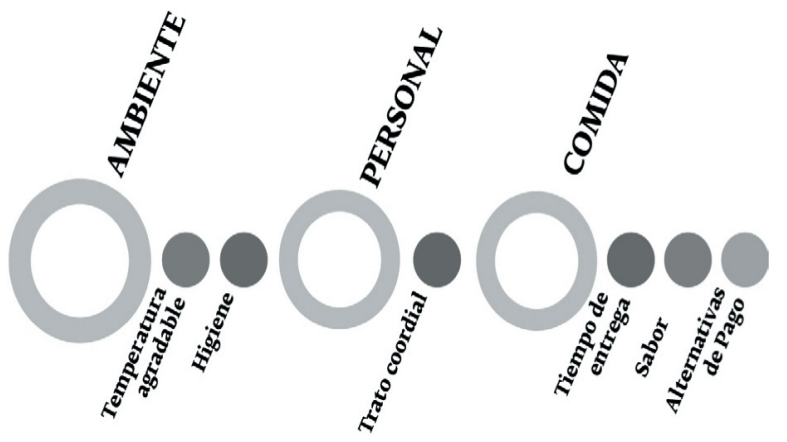

Figura 8. Factores claves del éxito para alcanzar la calidad en el servicio.

CONCLUSIONES

La investigación de mercados determinó que el cumplimiento y el buen servicio al cliente en esta clase de negocios se encuentran entre los tres factores más importantes para los 60 consumidores (18\%); siendo únicamente superado por la comida (29\%) y el ambiente del local (24\%). Esto coincide con Guerrón (2009), quien en su investigación concluye que la calidad y el servicio son un factor preponderante al seleccionar un restaurante.

Al momento de preguntar a los encuestados por el personal de un restaurante, respondieron que lo más importante para ellos es el trato cordial y la rapidez del servicio. Un dato que también se puede destacar es que el promedio más alto de los distintos grupos de variables lo obtuvieron los factores relacionados a la comida con una calificación 4,52 sobre 5 , seguido por el personal que ofrece el servicio en el restaurante, con una calificación de 4,03.

Como resultado final de las encuestas se puede concluir que los clientes que visitan restaurantes franquiciados en la ciudad de Cuayaquil pueden ser hombres y mujeres de todas las edades, siendo la mayoría entre los treinta y uno y cuarenta años de edad.

Por otro lado, los aspectos de mayor importancia según los encuestados fueron la limpieza y la adecuada temperatura que se percibe en estos locales, las diferentes alternativas de pago que se ofrece al cliente, y el trato cordial que se recibe por parte del personal de trabajo del local.

Y finalmente lo que espera el cliente ya sentado en su mesa, es que los pedidos sean entregados sin demoras y que los productos sean frescos y servidos a la temperatura adecuada según el plato solicitado.

\section{REFERENCIAS BIBLIOGRÁFICAS}

Guerrón Párraga, N. (2009): Plan de marketing estratégico para incrementar las ventas de la franquicia Tony Roma's ubicada en el Centro Comercial El Condado Shopping. (Tesis de pregrado, Universidad Politécnica del Ejército). Recuperado de: http://repositorio.espe.edu.ec/ handle/21000/2325.

INEC (2011). Avances del Censo Nacional Económico y Mecanismo de Difusión. Recuperado de: http://www.ecuadorencifras.gob.ec/

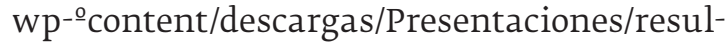
tados_generales_censo_economico.pdf

Palomeque, J. \& Ramírez, F. (2012): Perfil del mercado de franquicias en Ecuador. Quito: Cámara de Comercio Ecuatoriano Americana.

Redacción Guayaquil (2011, 17 de Octubre). 150 franquicias internacionales compiten en los 'malls' del país. El Comercio. Recuperado de: http://www.elcomercio.ec/ negocios/ franquicias-internacionales-compiten-malls-pais_0_573542751.html 